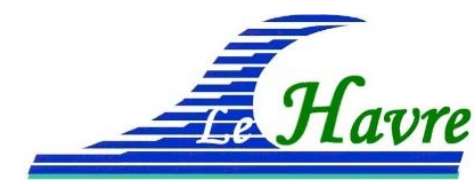

XVI İmes Journées Nationales Génie Côtier - Génie Civil

Le Havre, 2020

DOI:10.5150/jngcgc.2020.039 @ Editions Paralia CFL

disponible en ligne - http://www.paralia.fr - available online

\title{
Suivi participatif de l'érosion côtière en 3D : Demain tous SENTINELLES grâce au protocole SELPhCoAST
}

\author{
Elsa CARIOU ${ }^{1}$, Agnès BALTZER ${ }^{1}$, \\ Donatienne LEPAROUX ${ }^{2}$, Vincent LACOMBE ${ }^{3}$
}

1. Université de Nantes, UMR 6554 CNRS - LETG, Campus du Tertre, BP 81 227, 44312 Nantes Cedex 3, France.

elsa.cariou@univ-nantes.fr ; agnes.baltzer@univ-nantes.fr

2. Université Gustave Eiffel, Campus de Nantes, Allée des ponts et chaussées, CS 5004, 44344 Bouguenais Cedex, France.

donatienne.leparoux@univ-eiffel.fr

3. Société DIGISCAN3D, 21 chemin de la Fontaine, 85350 île d'Yeu, France.

v.lacombe@digiscan3d.fr

\section{Résumé :}

Dans le cadre du projet ODySéYeu, une réflexion collective (citoyens, associations, scientifiques, municipaux, privés) a été menée pour surveiller et suivre l'évolution de l'érosion littorale sur l'île d'Yeu (Vendée, France). Un cahier des charges basé sur les attentes des uns et des autres a été établi et les sociétés Studio-Matavai et DIGISCAN3D ont été mandatées pour mettre en place, avec l'équipe scientifique, une solution intégrée permettant de lever l'ensemble des verrous et mettre en œuvre le suivi de 9 sites sur l'île. Basés sur l'utilisation et l'optimisation de la technique appelée Structure from Motion by Smart devices photographies (SFM-S), le protocole SELPhCoAST et l'application pour smartphones/tablettes SENTINELLES sont le fruit de ce travail collectif. Cet article présente les résultats des 20 premiers mois de suivi sur le site de la plage de Ker Châlon. Il illustre l'efficacité de cette solution intégrée qui permet à l'ensemble des citoyens de devenir acteurs de la surveillance de l'érosion côtière et d'être mieux informés des enjeux et des risques. Cette solution offre aux scientifiques la possibilité de suivre l'évolution des sites en $3 \mathrm{D}$, à haute fréquence temporelle et à haute résolution spatiale. Elle permet aux décideurs de mettre en place des solutions de gestion du littoral parfaitement adaptées, en accord avec les citoyens-acteurs et "fondées sur la nature".

Mots-clés :

Erosion littorale, Suivi participatif en 3D, Smartphones et tablettes technologies, Structure from Motion-SfM, Solutions fondées sur la nature 


\section{Thème 3 - Instrumentation, mesures, imagerie et télédétection}

\section{Introduction}

Dans le contexte actuel de remontée du niveau marin, l'érosion côtière est un sujet de préoccupation majeure des territoires littoraux à échelle nationale comme mondiale. Le linéaire littoral à surveiller dépasse de très loin les capacités des professionnels seuls. De plus, les problématiques d'érosion littorale, en concernant à la fois les sphères économiques, sociales, politiques ou scientifiques, impliquent de facto des acteurs très divers, qui doivent pouvoir dialoguer et disposer d'outils et de corpus de connaissances communs. Ainsi, dans ce domaine, la "science collaborative" prend-elle tout son sens. S'appuyant sur la collaboration du grand public, elle offre des possibilités d'acquisitions à des fréquences bien supérieures aux capacités des professionnels seuls, permettant l'observation haute résolution de l'évolution du littoral.

Parmi les méthodes de surveillance utilisées pour suivre l'érosion côtière, la méthode photogrammétrique "Structure From Motion by Smart device photography" (SFM-S) est la méthode la plus prometteuse en termes de science collaborative (WESTOBY, 2018). En effet, elle allie l'aspect ludique de l'acquisition de photos côtières par smartphone et tablettes, à une qualité de données potentiellement proches des données professionnelles (quelques millimètres à quelques centimètres). Malgré son potentiel, l'emploi collaboratif de cette technique demeurait néanmoins, à ce jour, une "perspective", car quatre verrous majeurs s'opposaient encore à la mise en place d'une chaîne d'actions complète entre l'acquisition régulière des données de terrains par les acteurs locaux et l'usage des données interprétés par les collectivités, en matière de gestion littorale comme de communication (MICHELETTI et al., 2014 \& 2015 ; JAUD et al, 2019).

Ces verrous, détaillés ci-après, ont été levés grâce à l'approche développée au sein du projet ODySéYeu (Observation de la Dynamique Sédimentaire et environnementale autour de l'île d'Yeu). Cette dernière, à travers différentes phases de consultation, de tests et de validations, a permis la co-construction d'un nouvel outil intégré d'aide à la gestion littorale et au partage des savoirs à l'île d'Yeu, où neuf sites présentant des risques littoraux importants sont à présent suivis en $3 \mathrm{D}$ de manière collaborative (figure 1).

Le présent article propose ainsi la description méthodologique et les résultats de l'ultime phase de validation : le déploiement d'un premier suivi. 


\section{XVİ̀mes Journées Nationales Génie Côtier - Génie Civil \\ Le Havre 2020}
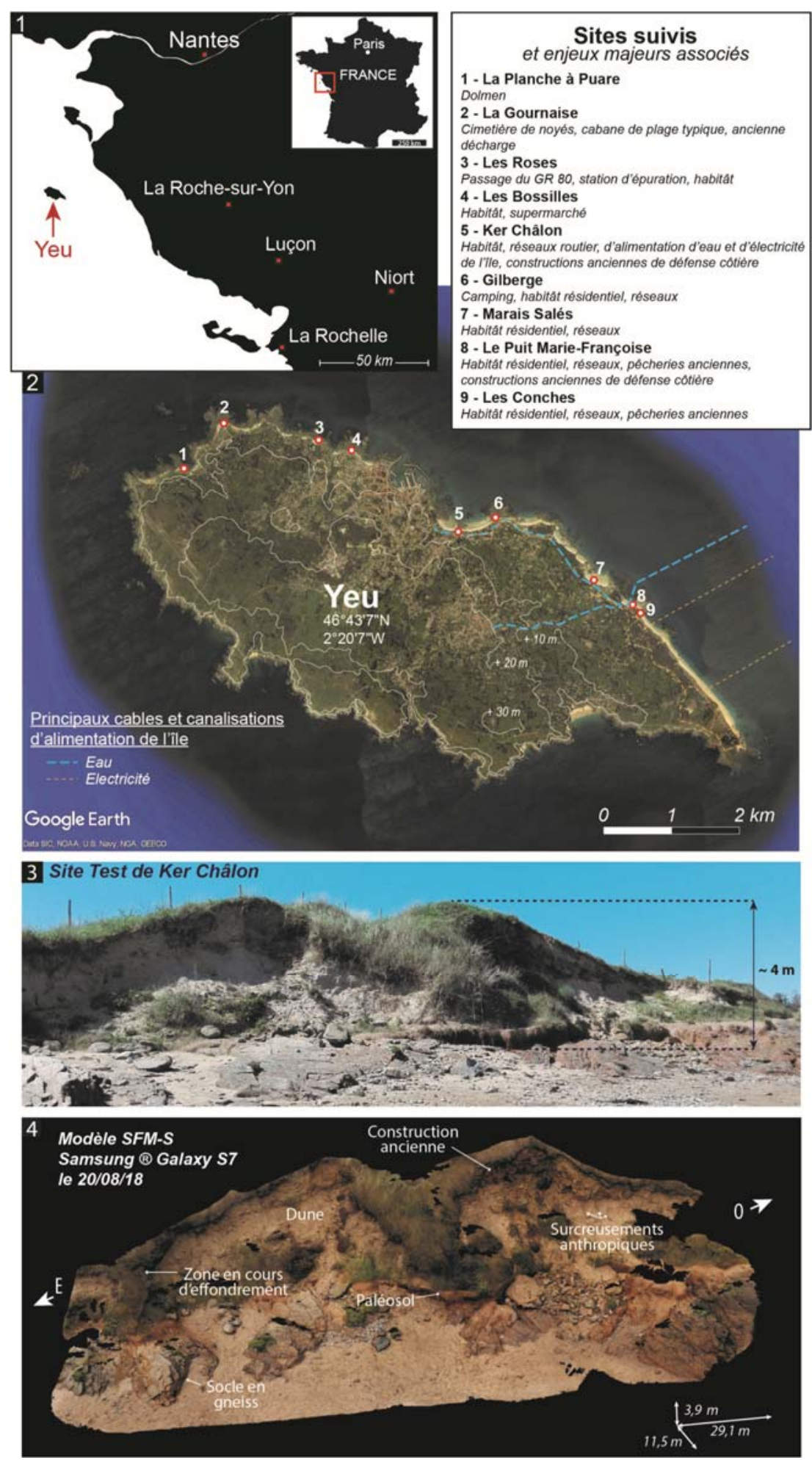

Figure 1. 1) Position géographique de l'île d'Yeu ; 2) localisation des sites suivis dans le cadre du projet ODySéYeu ; 3) Photographie du site de Ker Châlon en 2018 ; 4) Premier modèle SFM-S du site établi par l'intermédiaire d'un smartphone Samsung ${ }^{\circledR}$ Galaxy S7, le 20/08/18. 


\section{Thème 3 - Instrumentation, mesures, imagerie et télédétection}

\section{Verrous préexistants et démarche générale}

Le premier verrou concerne les informations connexes, nécessaires pour les corrections géométriques 3D. En effet, les modèles 3D reconstruits par SFM-S sont "sans dimension" et comportent potentiellement des déformations qu'il est important de pouvoir contrôler pour, le cas échéant, les corriger (WROZYNSKI et al., 2017). C'est pourquoi la plupart des tests scientifiques menés jusqu'à présent incluaient le renfort de matériel professionnel de type "Real Time Kinematics and Differencial GPS" (RTK DGPS), permettant d'acquérir un positionnement tridimensionnel plus précis que celui fourni par les puces GPS incluses dans les smartphones. Cet usage du DGPS requérait en outre l'usage de points de contrôle fixes appelés "Ground Control Points" (GCPs), généralement matérialisés par des objets contrastés et de forme préalablement caractérisée (JAUD et al., 2019). A l'instar du DGPS, matériel professionnel, par définition, incompatible avec un usage collaboratif, les GCPs classiquement utilisés posaient eux-mêmes le problème d'ajouts pérennes de "cibles" sur des portions littorales parfois classées. En effet, ces dernières constituent une pollution visuelle et suscitent la curiosité, entrainant potentiellement un risque de sur-dégradation. Il devient alors inapproprié de suivre de manière participative l'érosion d'une dune si la présence de cibles incite le public à la gravir pour voir les cibles de plus près. Le deuxième verrou vise l'aide à l'utilisateur. Cette notion requiert la capacité de guider précisément les participants dans l'acquisition des données, afin que la prise de donnée soit à la fois aisée et ludique pour l'usager, pertinente et complète pour le traitement, mais aussi optimale pour permettre une transmission fiable des données depuis l'appareil d'acquisition jusqu'à l'opérateur de traitement (CHIDBUREE et al., 2016). Parallèlement aux questions "de terrain", le troisième verrou repose sur le traitement des données par des techniques différentielles $3 \mathrm{D}$. Ces dernières requièrent des compétences scientifiques et techniques spécifiques, traditionnellement associées à des temps de calcul longs, peu compatibles avec l'afflux réguliers de données collaboratives (CHIDBUREE et al., 2016). Enfin, le bon usage de ces données, la communication et le partage des informations doivent être fluide entre les acteurs de terrain, les acteurs du traitement des données et les acteurs de la gestion littorale. Il est donc nécessaire de disposer d'outils de communication performants.

Afin de lever ces verrous et d'établir une chaine opérationnelle complète, le projet collaboratif ODySéYeu a invité l'ensemble de ses partenaires scientifiques, institutionnels, associatifs et privés locaux à établir la liste des besoins des usagers, qui permettrait le suivi collaboratif de la dynamique sédimentaire par SFM-S. Sur cette liste figurent notamment "simplicité d'acquisition et de transmission des données de terrain", "qualité des modèles 3D produits", "minimisation de l'impact visuel", et "facilitation du dialogue". A partir de cette liste, les sociétés partenaires DIGISCAN 3D (mesure 3D pour l'industrie et le patrimoine) et STUDIO MATAVAI (développement de solutions web), ont été mandatées pour proposer, un duo chaîne d'actions / application smartphone 


\section{XVIèmes Journées Nationales Génie Côtier - Génie Civil \\ Le Havre 2020}

satisfaisant à l'ensemble des exigences. Ainsi, la société DIGISCAN3D a-t-elle développé le protocole SELPhCoAST, qui inclue 1) un protocole simple permettant aux utilisateurs de terrain appelés "les Sentinelles" d'acquérir puis de transmettre, avec leur smartphone personnel, une série de clichés photographiques pertinents ;2) une solution de traitement des données basée sur la reconnaissance et l'usage de zones inertes telles des zones d'affleurements du socle rocheux ou des objets anthropiques fixés, permettant le recalage des modèles $3 \mathrm{D} ; 3$ ) une solution de diffusion des informations de terrain et des résultats du suivi permettant à tous les usagers d'être régulièrement informés.

Aux dates du 20/08/18 et 05/10/19, la mise en cuvre sur le terrain du protocole SELPhCoAST par une quinzaine d'utilisateurs néophytes, équipés de leurs propres smartphones, a permis de tester en conditions réelles la résolution et la répétabilité des résultats produits (CARIOU et al., 2020). L'emploi d'une quinzaine de modèles de smartphones différents a permis d'étendre les résultats préalablement obtenus par JAUD et al. (2019), et de confirmer l'indépendance entre modèle de smartphone et qualité des modèles 3D produits, pour tous les modèles de milieu et haut de gamme. Ces tests in situ ont également montré la capacité de la chaîne d'action à produire des modèles fidèles aux modèles acquis de manière professionnels avec un TLS (Terrestrial Laser Scanner), dans une gamme de tolérance de 2 écart-types ( $=95 \%$ des points) à moins de $5 \mathrm{~cm}$ de la référence. L'expérience montre même que dans la plupart des cas ces exigences sont remplies pour plus de $99 \%$ des points (voir exemples en figures 2 et 3 ).

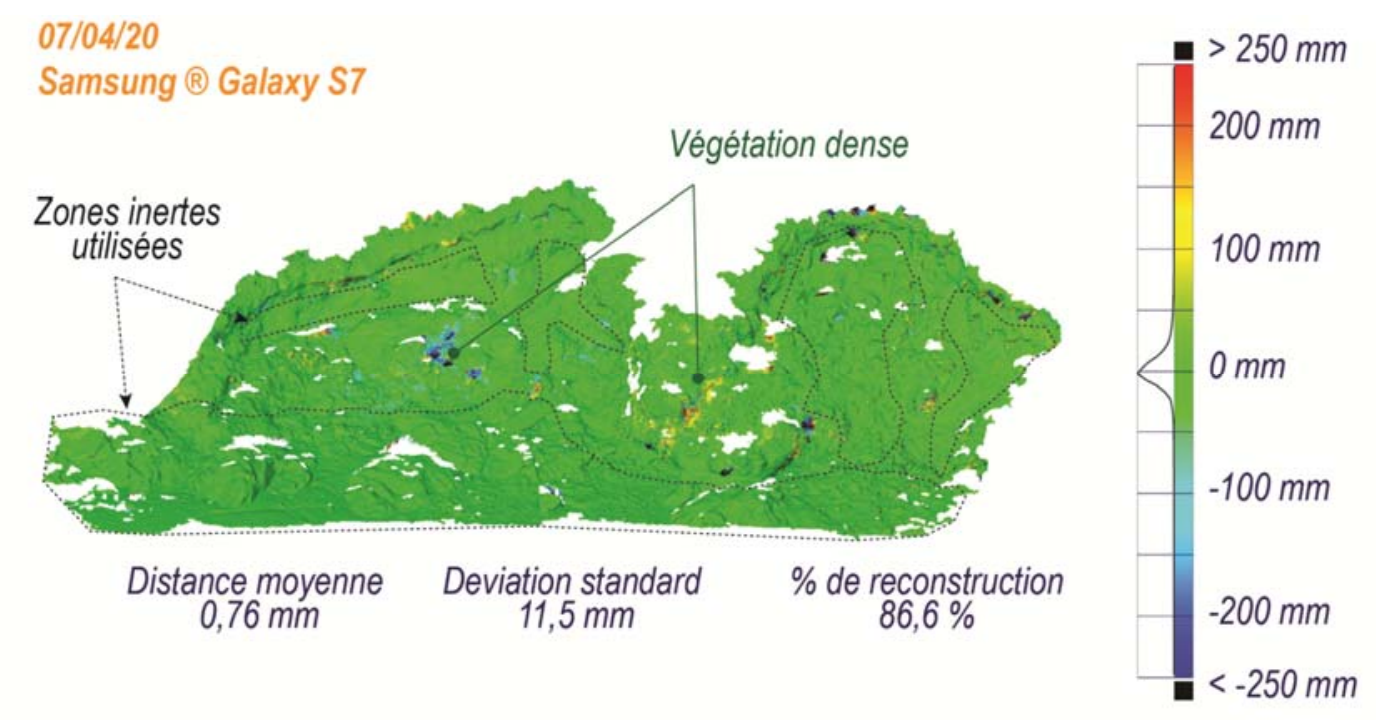

Figure 2. Distribution spatiale des différences entre les modèle SFM-S et TLS du 07/04/20. Dist. moy, dév. stand. et \% de rec. ont été calculés sur les zones considérées inertes en prenant le modèle TLS comme référence. L'histogramme de répartition des points, adjacent à l'échelle colorimétrique, a été calculé pour l'ensemble du modèle

(figure adaptée de CARIOU et al., 2020). 


\section{Thème 3 - Instrumentation, mesures, imagerie et télédétection}

Pour finaliser la validation de l'ensemble de la chaine d'action, la dernière étape "test" consiste à quantifier la répétabilité des résultats produits. Dans ce but, le suivi régulier d'un site "test" a été mis en place sur 20 mois du 20/08/18 au 07/04/20. Le présent article décrit cette dernière étude menée sur le site test de Ker Châlon (île d'Yeu, Vendée, France), suivant le protocole d'acquisition établi dans SELPhCoAST, à l'aide de smartphones individuels (Samsung ${ }^{\circledR}$ Galaxy S7 and a Huaiwei ${ }^{\circledR}$ P20 Pro).

\section{Zone d'étude}

Le site de Ker Châlon (figure 1.2-4) a été choisi pour le premier suivi-test, car il présente la morphologie la plus complexe (figure 1.3-4), avec deux lobes d'érosion distincts et profonds (5 à $11 \mathrm{~m})$, et de nombreux éboulis. Ceci permet donc de tester la fiabilité des reconstructions dans les trois dimensions de l'espace, et l'impact de la présence de nombreux contre-champs. Ker Châlon est également un excellent exemple de site où les enjeux côtiers s'entremêlent. La zone suivie est située sur une plage extrêmement fréquentée et, aux grandes marées, la mer atteint le pied de falaise dunaire. Elle provoque d'importants mouvements de sable entre les zones d'affleurement du socle de gneiss et vient lécher le paléosol qui le recouvre (figure 1). Ce paléosol est recouvert d'une dune d'environ $2 \mathrm{~m}$, elle-même coiffée d'une construction en pavés taillés. D'après les investigations menées aux côtés du service patrimonial municipal, il semblerait que cette ruine soit le vestige de la première série de batteries de défense côtière édifiées sur l'île au $17^{\text {ème }} \mathrm{s}$. A l'heure actuelle, le relief positif de ce site protège de la mer un bâtiment récent, un parking et la principale conduite d'alimentation en eau de l'île, ainsi qu'une dizaine d'habitations, dans un rayon de $100 \mathrm{~m}$.

\section{Matériel et méthodes}

Trois campagnes annuelles d'acquisition de données au TLS (Terrestrial Laser Scanner) ont été réalisées sur la période de tests de 20 mois (20/08/18, 14/05/19 et 07/04/20). Les modèles issus de ces campagnes ont été inclus dans la chronologie générale des modèles réalisés. Le TLS utilisé est un FARO ${ }^{\circledR}$ Focus $S 70$ qui scanne dans une sphère de $70 \mathrm{~m}$ à $360^{\circ}$ horizontalement et à $270^{\circ}$ verticalement, avec une erreur de recalage inférieure à 1 $\mathrm{mm}$, à $10 \mathrm{~m}$ de la source et inférieure à $3,5 \mathrm{~mm}$ à $25 \mathrm{~m}$. Dix stations ont été nécessaires à la réalisation d'un nuage de points décrivant l'ensemble du site et pour la réalisation du nuage de points final (88,4 millions de points), seuls les points scannés à moins de $25 \mathrm{~m}$ des stations ont été pris en compte. Généré par le logiciel FARO® SCENE 2018, ce nuage de points a ensuite été importé dans 3DReshaper® 2017, et converti en un maillage de plusieurs millions de polygones. Le modèle produit en 2018 est le modèle de référence sur lequel tous les autres modèles (TLS et SFM-S) ont été réalignés.

L'essentiel du suivi présenté ici a été réalisé grâce au traitement de séries de 33 clichés photographiques, régulièrement acquis, en suivant rigoureusement la chaîne d'action SELPHCoAST. Deux smartphones de modèles Samsung ${ }^{\circledR}$ Galaxy S7 et Huaiwei ${ }^{\circledR}$ P20 


\section{XVİ̀mes Journées Nationales Génie Côtier - Génie Civil \\ Le Havre 2020}

Pro ont été utilisés. Toute intelligence artificielle inclue dans les deux appareils et susceptible de prétraiter les clichés en amont de leur enregistrement sur la carte SD a été préalablement désactivée.

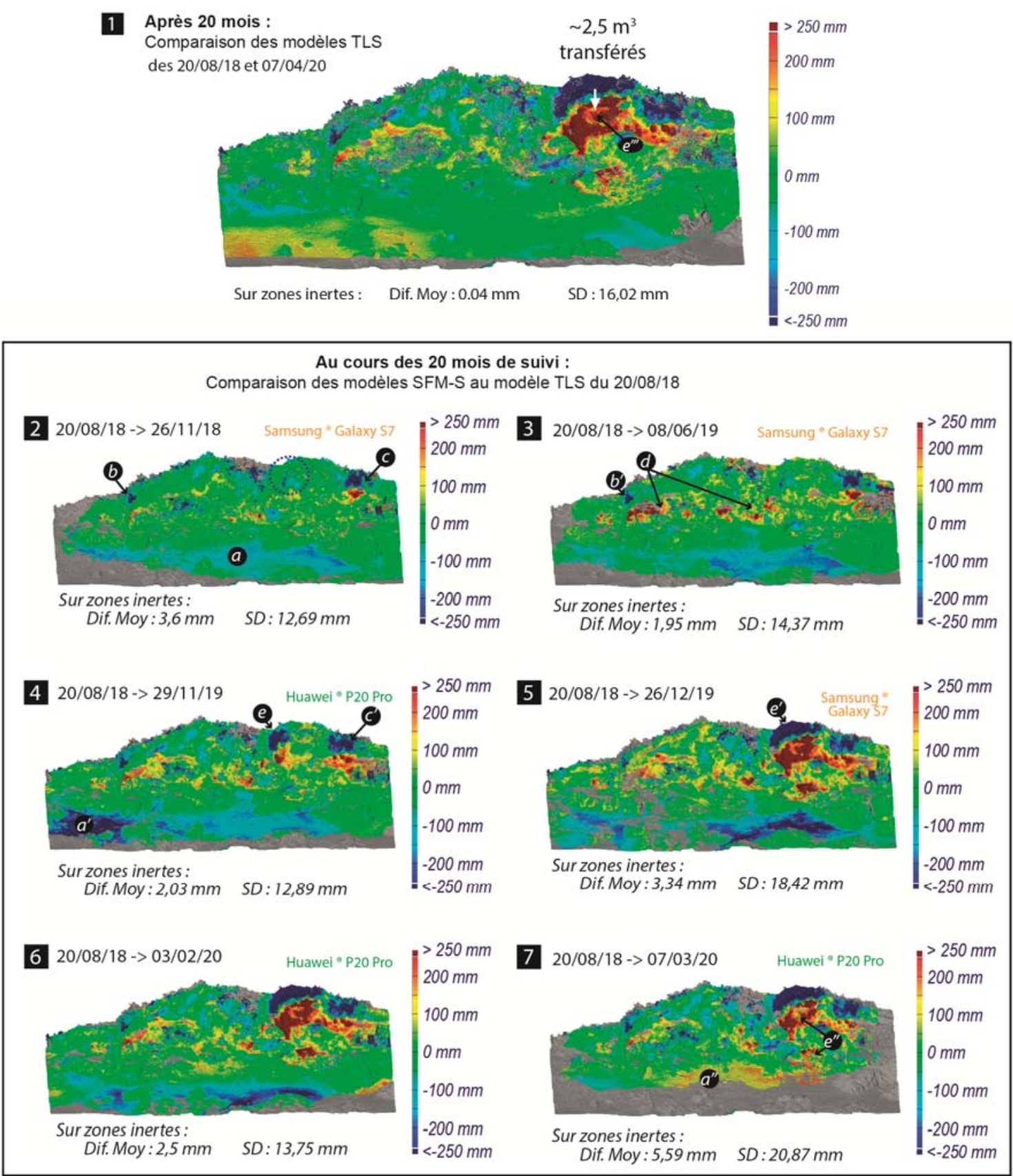

Figure 3. Répartition spatiale des différences mesurées (en $\mathrm{mm}$ ) pour chaque modèle reconstruit au cours du suivi de 20 mois (qu'il soit issu de données TLS ou smartphones), par comparaison au modèle TLS de référence du 20/08/18. Les zones signalées par de petites lettres sur fonds noir font référence au texte et aux événements de la frise chronologique en figure 4. 


\section{Thème 3 - Instrumentation, mesures, imagerie et télédétection}

Les clichés obtenus ont des résolutions de 4332x3024 Pix et 3648x2736 Pix. Les zones du site où affleure le socle en gneiss ont été utilisées pour recaler les modèles entre eux. Sur ces mêmes zones prédéfinies les distances moyennes au modèle TLS de référence (2018) et les déviations standards associées ont été calculées à l'aide du logiciel GOM Inspect ${ }^{\circledR}$ Pro. Les zones de végétation sont repérées sur les modèles texturés et ne sont pas prises en compte lors de l'analyse des comparaisons entre modèles.

\section{Résultats et interprétation du suivi}

La figure 3 montre la répartition spatiale des écarts au modèle de référence calculés grâce aux différents modèles TLS et SFM-S reconstruits au cours de la période test de 20 mois. Sur les zones considérées inertes (gneiss), les écarts à la référence moyens constatés varient entre $5,6 \mathrm{~mm}$ et $1,95 \mathrm{~mm}$ pour les modèles SFM-S, contre $0.04 \mathrm{~mm}$ pour le modèle TLS. Relativement comparables à celles du modèle TLS (16.02 mm), les déviations standard des modèles SFM-S oscillent quant à elles entre $20,87 \mathrm{~mm}$ et $12,69 \mathrm{~mm}$ sur ces mêmes zones. Ces gammes de valeurs de quelques millimètres, très faibles à l'échelle du site, retrouvées à chaque nouvelle acquisition, confirment la justesse des reconstructions 3D réalisées via le protocole SELPhCoAST (CARIOU et al., 2020) pour l'ensemble des tests réalisés.

Après 20 mois, la comparaison des modèles TLS initiaux et finaux (du 20/08/18 et du 07/04/20) montre que, sur l'ensemble du site, l'érosion ne s'est pas distribuée de façon homogène (figure 3.1). Les écarts au modèle de référence s'étalent de $+350 \mathrm{~mm}$ (zones pourpres sur le modèle) à $-1200 \mathrm{~mm}$ (zones en bleu foncé), et montrent une évolution différentielle de la morphologie du site, principalement concentrée dans sa partie occidentale (à droite sur le modèle) au niveau des zones sub-verticales de falaise et pied de falaise. Les zones subhorizontales du site semblent quant à elles avoir très peu évolué, suggérant une dynamique faible du haut de plage. Cette seule comparaison, faisant le bilan de l'ensemble des événements subis par le site sur 20 mois permet d'identifier les mouvements majeurs, et d'estimer à $2,5 \mathrm{~m}^{3}$ la quantité de matériel effondré dans la zone occidentale. Elle ne permet pas en revanche de déterminer l'origine ou la temporalité des mouvements. En outre, elle masque totalement la dynamique à haute fréquence du haut de plage. En effet, au cours de cette période de 20 mois, l'acquisition participative de clichés smartphone, en suivant le protocole SELPhCoAST, a permis de compléter la série temporelle (ensemble de la figure 3) par 6 modèles supplémentaires. La série complète révèle la dynamique du sable de haut de plage (a sur la figure 3.2) avec la formation éphémère de creux (jusqu'à $-300 \mathrm{~mm}$ sur le modèle de référence, a' sur la figure 3.4) ou de bosses (jusqu'à $+170 \mathrm{~mm}$, a" sur la figure 3.7) de sable entre les zones de socle. La série temporelle et les observations des opérateurs de terrain ont également permis de préciser la temporalité des mouvements de terrain observés et d'identifier leur source avec plus de précision. Par exemple, sur la partie orientale du modèle de novembre 2018 (figure 3.2), un couple "bleu au-dessus/rouge en dessous", repérée par un b sur le modèle, 


\section{XVI'̀mes Journées Nationales Génie Côtier - Génie Civil \\ Le Havre 2020}

pointe typiquement un premier effondrement dans la partie orientale du site. L'observation rétrospective du modèle texturé d'août 2018 (figure 1) montre que cette zone avait alors déjà commencé à s'effondrer (présence de matériel éboulé à la base). L'observation des modèles postérieurs à novembre 2018 (figure 3) montre que cet effondrement ne s'est pas amplifié après novembre 2018. Toujours sur le modèle de novembre 2018, un second effondrement plus important est observé dans la partie occidentale du site (c sur la figure 3.2). A cet endroit, lors du scan TLS du 20/08/18, trois creusements arrondis, profonds d'une vingtaine de centimètre (visibles sur la figure 1) avaient été observés. Les participants volontaires $\mathrm{du}$ test participatif réalisé simultanément au scan TLS, avaient alors signalé que ces dépressions avaient été creusées 6 jours auparavant, par un groupe de personnes souhaitant s'installer confortablement pour observer un feu d'artifice. Le modèle de novembre 2018 montre donc qu'en quelques mois, ces "creux" avaient déjà été comblés par l'effondrement du matériel initialement situé juste au-dessus. Entre juin et novembre 2019, l'extension de la zone impactée s'est agrandi (c'sur la figure 3.4), montrant ainsi les conséquences à retardement de l'action humaine. A partir de novembre 2019, une nouvelle zone d'effondrement semble brutalement apparaître sur les modèles, à quelques mètres à gauche de la précédente (e sur la figure 3.4). Malgré leur proximité, les deux effondrements ne semblent pas génétiquement liés. En revanche, une analyse rétrospective des modèles antérieurs montre que la déstabilisation de cette zone de surplomb était probablement discrètement amorcée (zone entourée en pointillés sur la figure 3.2), a minima dès la fin de l'été 2018. Au même endroit, le modèle du 26/12/19 enregistre l'accélération du processus d'effondrement, avec plusieurs mètres linéaires subitement engagés (e' sur la figure 3.4). Sur le modèle du 07/03/20, le matériel effondré dans cette zone a été en partie dispersé. Le 07/04/20, le matériel dispersé a été remplacé par du matériel "frais", issu d'un nouvel épisode d'effondrement, montrant que, malgré un ralentissement du processus, la zone demeure très instable. Le matériel effondré est essentiellement composé de blocs de pierre issus des ruines qui coiffent le site. La mise en perspective des mouvements de terrain observés avec la chronologie des tempêtes majeures sur la même période (figure 4) montre que ces dernières semblent très impliquées dans la dynamique morphologique du site. Les mouvements du sable de haut de plage observés, montrent l'action régulière de la mer sur la partie inférieure du site. Contre toute attente, la période hivernale 2019-2020, riche en tempêtes (Ciara, Denis et Léon), semble plutôt avoir favorisé l'engraissement de la plage au pied de la zone érodée. Si l'action de la mer pendant ces tempêtes a pu participer à la dispersion du matériel préalablement effondré, elle ne semble pas non plus avoir provoqué de surcreusement dans la partie basale du site. En revanche, la tempête Fabien, puis les pluies diluviennes de début mars 2020, suivies de vents d'est très violents, semblent avoir nettement facilité l'effondrement majeur "e". A l'échelle de ce suivi de 20 mois, ce sont donc clairement l'activité humaine, la pluie et le vent qui ont provoqué la dégradation du site, et non la mer. 


\section{Thème 3 - Instrumentation, mesures, imagerie et télédétection}

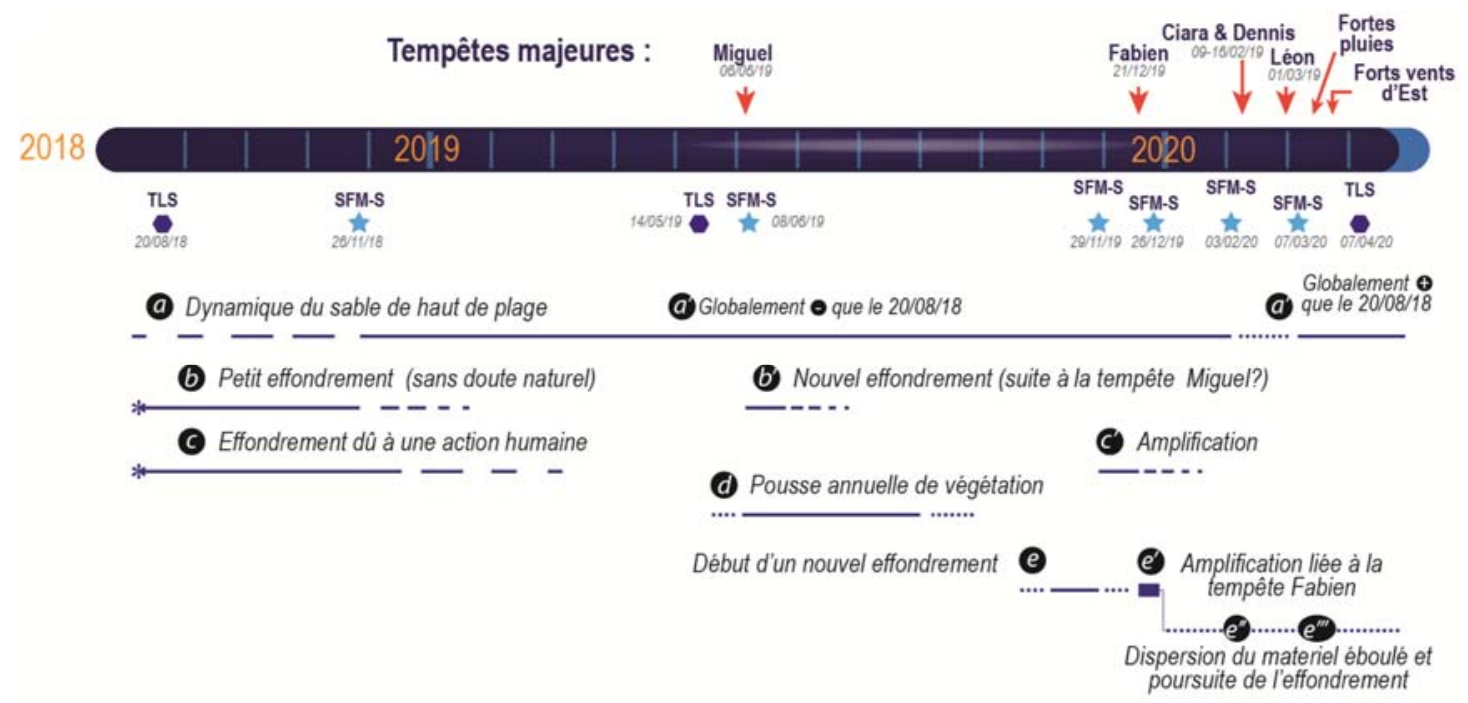

Figure 4. Frise chronologique reconstruite à partir du croisement des informations de terrain et de l'observation des modèles issus du suivi participatif via SELPhCoAST.

Ainsi, les 20 mois de suivi participatif de ce site montrent-ils l'efficacité potentielle de ce type de suivi pour adapter les politiques de gestion à mener sur chaque site. A cet endroit, la mise en place d'un enrochement à la base du site (demandé à la municipalité par certains riverains) serait très probablement source de dégâts supplémentaires, en générant des turbulences hydrodynamiques nouvelles. En revanche, empêcher totalement l'accès au site semble urgent, aussi bien pour la sécurité des personnes que pour la préservation du site. Compte-tenu de la taille du site, des volumes érodés et de vitesses de recul, mener une réflexion sur le devenir de la zone et en particulier sur la politique à mener vis-à-vis des vestiges patrimoniaux qu'elle contient semble indispensable.

L'implication de dizaines de citoyens dans le processus de suivi à l'île d'Yeu, et la possibilité pour eux de transmettre facilement des photographies et des observations de terrain à travers l'application SENTINELLES, permettra d'établir chaque mois un nouveau modèle 3D, d'une très haute résolution, pour chacun des sites identifiés dans le projet. Il a été choisi dans le protocole SELPhCoAST de rendre accessible au public l'ensemble des modèles colorés et des modèles de comparaison réalisés, par l'intermédiaire de la plateforme Sketchfab ${ }^{\circledR}$. Cette plateforme offre aux structures de recherche et de conservation la possibilité de mettre en ligne des modèles 3D et d'y adjoindre des informations complémentaires, comme par exemple de courts textes ou des images. Ainsi, l'intégralité du suivi (interprétations comprises) pourra-elle être partagée en ligne par l'ensemble des acteurs, qu'ils soient citoyens, scientifiques ou institutionnels. 


\section{XVI'̀mes Journées Nationales Génie Côtier - Génie Civil \\ Le Havre 2020}

\section{Conclusion}

Le protocole SELPhCoAST et l'application SENTINELLES ont été développés pour permettre la mise en place d'un suivi participatif de l'érosion côtière sur l'île d'Yeu. Le travail de développement réalisé a permis de s'affranchir totalement de cibles et d'optimiser l'acquisition puis la transmission de photos smartphones, par des opérateurs de terrain inexpérimentés (y compris des enfants). Le suivi-test présenté ici, réalisé sur une durée de 20 mois, met en évidence la capacité et la pertinence de ce protocole pour construire des modèles 3D complets et très fiables des sites, sur la seule base des photos acquises de manière participative. Ainsi, le suivi à haute fréquence et haute résolution de ces sites permet de décrypter précisément la dynamique et les phénomènes responsables des mouvements de terrain. Pour chaque site il sera alors possible de mener des actions de protection sur-mesure, "fondées sur la nature", et s'appuyant sur une démarche d'observation collaborative. Ces actions auront alors d'autant plus de crédit auprès des acteurs, qu'ils soient scientifiques, décisionnaires ou citoyen concerné et investi.

\section{Références bibliographiques}

CHIDBUREE P., MILLS J. P., MILLER P. E., FIEBER K. D. (2016). Towards a lowcost real-time photogrammetric landslide monitoring system utilizing mobile and cloud computing technology, International Archives of the Photogrammetry, Remote Sensing and Spatial Information Sciences, Vol. XLI-B5, pp 791-797, https://doi.org/10.5194/isprsarchives-XLI-B5-791-2016

CARIOU E., BALTZER A., LEPAROUX D., JAUD M., LACOMBE V. (2020). Structure from motion by smart-devices photography (SfM-S) for participatory monitoring projects: validation of the SELPhCoAST workflow and its results. Remote Sensing. paper submitted.

JAUD M., KERVOT M., DELACOURT C., BERTIN S., (2019). Potential of smartphone SfM photogrammetry to measure coastal morphodynamics. Remote Sensing, MDPI, Vol. 11, 2242, https://doi.org/10.3390/rs11192242

MICHELETTI N., CHANDLER J.H., LANE S.N. (2014). Investigating the geomorphological potential of freely available and accessible structure-from-motion photogrammetry using a smartphone. Earth Surf. Process. Landforms, Vol. 40 (4), pp 473-486. http://dx.doi.org/10.1002/esp.3648

MICHELETTI N., CHANDLER J.H., LANE S.N. (2015). Structure from motion (SfM) photogrammetry, Geomorphological techniques, British society for Geomorphology, Chap.2, Sec 2.2. 
Thème 3 - Instrumentation, mesures, imagerie et télédétection

WESTOBY M.J., LIM M., HOGG M., POUND M.J., DUNLOP L., WOODWARD J. (2018). Cost-effective erosion monitoring of coastal cliffs. Coastal Engineering, Vol. 138, pp 152-164. https://doi.org/10.1016/j.coastaleng.2018.04.008

WRÓŻYŃSKI R., PYSZNY K., PRZYBYŁA C., MURAT-BŁAŻEJEWSKA S. (2017). Ground volume assessment using 'Structure from Motion' photogrammetry with a smartphone and a compact camera. Open Geosciences, Vol. 9, pp 281-294. https://doi.org/10.1515/geo-2017-0023 